\title{
Visualizing Chemical Dynamics in an Ionic Liquid Microdroplet using Ultrafast 2D IR Microscopy
}

Kathryn M. Tracył, Biswajit Guchhait ;, Clara A. Tibbetts, Bradley M. Luther, Amber T.

Krummel*

Department of Chemistry, Colorado State University, Fort Collins, Colorado, 80523-1872, United States

KEYWORDS Ionic Liquids, Microdroplet, 2D IR Microscopy, 2D IR Spectroscopy, Ultrafast Dynamics

ABSTRACT. Chemical dynamics in a room temperature ionic liquid (RTIL) microdroplet are revealed with two-dimensional infrared (2D IR) microscopy performed with fast acquisition rates. Herein we report the first demonstration of high repetition rate 2D IR microscopy applied to a complex chemical system. Spatially resolving the time-dependent 2D IR signals using tricyanomethanide (TCM-) as a vibrational probe exhibits three regions within the RTIL microdroplet with different chemical dynamics — the bulk, the interface, and intermediate regions. The spectral diffusion behavior of TCM- shows that structural relaxation at the microdroplet interface is significantly slower than the bulk or interior environment. Quantifying the chemical dynamics within the RTIL microdroplet provides insights that pave the way towards understanding 
the heterogeneous nature of dynamic behaviors in RTILs. Moreover, we demonstrate the potential power of 2D IR microscopy to reveal chemical dynamics in complex environments, which remain hidden in other chemical imaging approaches, including FTIR, CARS, SFG, and SHG microscopy.

\section{Table of Contents Figure}

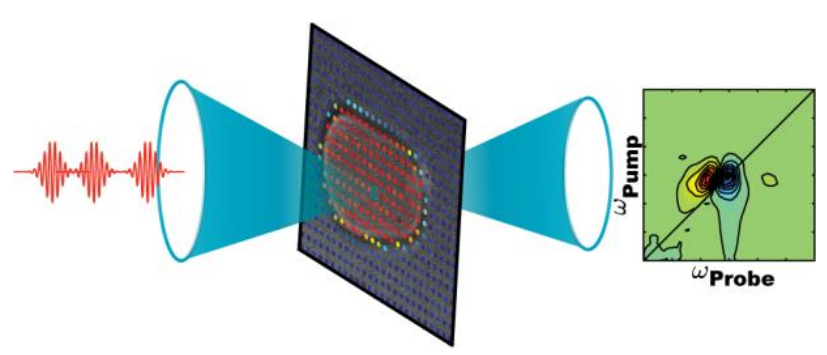

\section{Introduction.}

Recently, microemulsion and microdroplet technologies have received considerable attention because of their striking ability to confine a reaction in a very small volume ( $\mu \mathrm{L}$ or $\mathrm{nL})$ and thus making previously difficult chemical reactions more favorable.1-6 Chemical reactions, such as acid catalyzed synthesis of isoquinoline, in microdroplets can be accelerated by more than 106 times in microdroplets in comparison to bulk reaction conditions.2 It has also been observed that phosphorylation of sugar, a thermodynamically unfavorable reaction in bulk phase, becomes favorable in microdroplets in the absence of any enzyme.5 In addition, many researchers have sought to compartmentalize room temperature ionic liquids (RTILs) as designer fluids in droplet microfluidics7-10 and have found several important advantages of using RTILs as solvents when compared with organic liquids. They are low melting salts with unique properties such as having a wide electrochemical window, an extremely low vapor pressure, and structural 
microheterogeneity.11-13 These properties make RTILs strong candidates for a wide variety of applications from biotechnology14 to energy technology,15-17 including as reaction media.18,19

RTILs can be tailored to specific purposes due to the ease of interchanging the counter ions involved, however, this is done in large part using empirical evidence, rather than having the ability to predict the behavior of RTILs. Therefore, it is crucial to gain insights into the fundamental aspects of interactions within RTILs including structural dynamics, solute-solvent interactions, and solvation dynamics. Recently, investigators have turned to nonlinear optical spectroscopy techniques including sum frequency generation spectroscopy 20 and two-dimensional infrared (2D IR) spectroscopy21-26 to characterize RTILs at interfaces and measure the dynamics that occur in bulk RTIL environments, respectively. In order to advance the utility of RTILs in microdroplet chemistry, the length scales over which various dynamical behaviors exist are important.23,27-31

Although acceleration of reaction rates and many types of previously unknown reactions have been observed in miniaturized microdroplets, the physical mechanism of the acceleration phenomenon is not yet known. It has been proposed that a different chemical behavior of reactant molecules at the surface of the microdroplet may speed up and favor a reaction. However, the fundamental aspects of structure, ultrafast dynamics, and solvation, which are crucially important determinants in regulating reaction rates in such microdroplets, are entirely unknown. In this work, we characterize ultrafast structural dynamics of a RTIL microdroplet using 2D IR microscopy. Microdroplet chemistry is an emerging field and the present 2D IR imaging studies of a RTIL microdroplet illuminate details of dynamics and long-range effects, which pave the way for a more detailed exploration of this new field.

Recent efforts have focused on spatially resolving molecular structure and dynamics of heterogeneous samples and important experiments demonstrating the feasibility of ultrafast 2D IR 
microscopy have been performed.32,33 A major impediment to extensive practical implementation of 2D IR microscopy is the long acquisition time. To improve imaging efficiency, we have moved away from the traditional Ti:sapphire based laser and optical parametric amplification (OPA) systems that operate at one to a few $\mathrm{kHz}$ repetition rates. Recently we demonstrated high throughput 2D IR spectroscopy utilizing a mid-IR optical parametric chirp pulse amplifier (OPCPA) laser system operating at a $100 \mathrm{kHz}$ repetition rate.34,35 In order to study the structural dynamics in a microdroplet, we perform 2D IR microscopy utilizing our Yb-based, $100 \mathrm{kHz}$ midIR OPCPA laser source. Taking advantage of the high repetition rate and improved signal-tonoise ratios over Ti:sapphire laser sources, 2D IR microscopy experiments are carried out with much faster acquisition rates. Here we have studied a microdroplet formed with the RTIL, 1-ethyl3-methylimidazolium tetrafluoroborate $\left(\mathrm{EmimBF}_{4}\right)$ doped with the vibrational probe, tricyanomethanide (TCM-). Spatially resolving the time-dependent 2D IR signals reveals distinctly different dynamic behavior, including differences in homogeneous and inhomogeneous contributions to the spectral line shape at the bulk and interface of the RTIL microdroplet. In addition, intermediate regions between the interface and bulk regions are investigated.

\section{Experimental Methods.}

Sample Preparation. The samples utilized in both the 2D IR and FTIR microscopy experiments were RTIL microdroplets in silicon oil. The droplets consisted of 1-ethyl-3-methylimidazolium tetrafluoroborate $(\mathrm{EmimBF} 4)$ and 1-ethyl-3-methylimidazolium tricyanomethanide (EmimTCM) RTILs. The EmimTCM is used as a vibrational probe in these experiments and is doped into the EmimBF4 in a 1:500 volume ratio. This volumetric ratio corresponds to one EmimTCM ion pair in 606 EmimBF4 ion pairs. The dimethyl silicone oil was obtained from Thomas Scientific, 
SF96/50. EmimBF4 and EmimTCM (Iolitec) were dried under vacuum for 24 hours. All experiments were performed at room temperature; the samples were placed between two $\mathrm{CaF}_{2}$ plates using a 100- $\mu \mathrm{m}$ thick Teflon spacer for FTIR and 2D IR imaging experiments. A roughly spherical droplet is formed when several nanoliters of RTIL $(10-20 \mathrm{~nL})$ is casted in silicon oil. The droplet becomes a pancake in shape when placed in between two $\mathrm{CaF}_{2}$ substrates. The oil is used for stabilization of the RTIL microdroplet as well as removing scatter from the interface because the refractive index of the silicon oil is similar to the RTIL microdroplet. In addition, the silicon oil preferentially wets the $\mathrm{CaF}_{2}$ plates; therefore the RTIL microdroplet is surrounded on all sides by the silicon oil.

FTIR Imaging. A Bruker Hyperion 3000 FTIR microscope was used to collect the FTIR chemical image. The FTIR microscope utilizes a 64 x 64 element FPA detector and a 15x objective to give a $7.6 \times 7.6 \mu \mathrm{m} 2$ spatial resolution. The FTIR microscope image was taken in transmission mode and was processed using OPUS software (Bruker Optics).

2D IR Imaging. 2D IR microscopy was performed with a $100 \mathrm{kHz}$, mid-IR source based on optical parametric chirped-pulse amplification (OPCPA) in magnesium doped periodically poled lithium niobate (MgO:PPLN) and difference frequency generation (DFG) in zinc germanium diphosphide (ZGP), which generates $3 \mu \mathrm{J}$ of $4.65 \mu \mathrm{m}$ mid-IR output with $220 \mathrm{fs}$ pulse durations. This source has been described in previous work. 34

The 2D IR microscope is detailed in Figure 1. The IR output is split into two lines using a $\lambda / 2$ waveplate and wire grid polarizer. The polarizer reflects the probe pulse whose polarization is rotated by $90^{\circ}$, creating S-polarization. The P-polarized pump line passes through the polarizer and is sent to a high speed, mid-IR pulse shaper to generate the pump pulse pair with a variable delay at $100 \mathrm{kHz}$. The pump and probe pulses are recombined using a polarizer that transmits the 
pump pulses and reflects the probe pulse to create a collinear geometry where 2D IR data is collected in the cross polarization (XXYY) configuration. The three pulses are focused into the sample by a $\mathrm{Ge} / \mathrm{Si}$ achromatic lens and then re-collimated using a Si lens and sent to the detector. The spot size was measured by scanning a $5 \mu \mathrm{m}$ pinhole in the $\mathrm{X}$ and $\mathrm{Y}$ direction at the focal plane and recording the integrated intensity. The full width at half maximum (FWHM) beam diameter was measured to be $19.5 \mu \mathrm{m}$ in the $\mathrm{Y}$ direction and $14.2 \mu \mathrm{m}$ in the $\mathrm{X}$ direction. The shape of the third order signal emitted from the sample given the shape of the pump and probe beams is shown in Figure 1b; the FWHM beam diameter of the third order signal is $11.4 \mu \mathrm{m}$. The Rayleigh range of focus in the direction of propagation is approximately $140 \mu \mathrm{m}$. The two polarizers before the detector are used to block the pump pulses and transmit the probe and signal. The probe and signal fields are then passed through a monochromator and collected on a 1x64 element, mercury cadmium telluride (MCT) linear array detector operating at the speed limit of detection of 100 kHz. A 100 lines/mm grating was used in the monochromator. Considering the physical dimensions of the monochromator, MCT element dimensions, and the gratings, the resolution along the probe axis ( $\omega$ probe) of the 2D IR spectra collected is $3.3 \mathrm{~cm}-1$. 

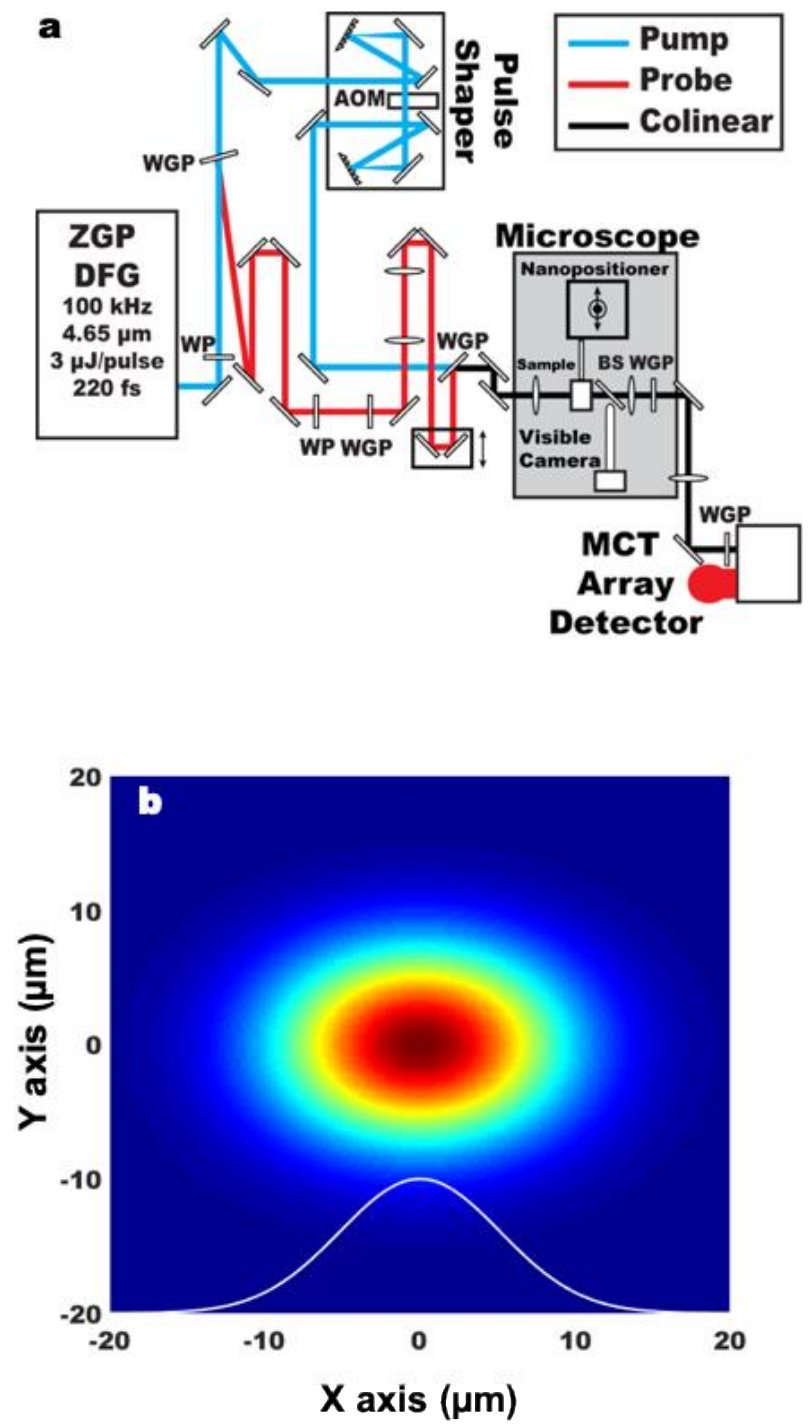

Figure 1. Block Diagram of the $100 \mathrm{kHz}$ repetition rate 2D IR microscope (a). Calculated shape of the third order signal emitted given the spatial dimensions of the pump and probe beams impinging the sample (b).

The 2D IR spectra were all collected using a series of 510 pump pulse pairs delayed from 0 to $3.57 \mathrm{ps}$ in $7 \mathrm{fs}$ steps. Thus, the spectral resolution along the pump-axis ( $\omega$ pump) is $4.7 \mathrm{~cm}-1$. Spectra were acquired using a four-step phase cycling scheme with $2000 \mathrm{~cm}-1$ rotating frame for background and scatter removal. The full 2D IR microscopy image of the RTIL microdroplet, 
shown in Figure 3a, is comprised of a data set of 476 2D IR spectra fully averaged 500 times. The pulse energies utilized were $70 \mathrm{~nJ} /$ pulse.

The sample was held in a lens mount, which has been mounted to a 2-axis translational stage. The adjustment of the focus on the sample in the direction of propagation is obtained by controlling the $\mathrm{Ge} / \mathrm{Si}$ lens position with a manual stage. The sample position across the focusing area in the $\mathrm{X}$ and $\mathrm{Y}$ direction is controlled by a nanopositioner and two micropositioners (Nano-3D200 and MMP, Mad City Labs Inc.) with a $200 \mu \mathrm{m}$ range of motion and a $25 \mathrm{~mm}$ range of motion, respectively. To collect the $450 \mu \mathrm{m}$ x $600 \mu \mathrm{m}$ image seen in Figure 2a, $25 \mu \mathrm{m}$ steps were taken in the $\mathrm{X}$ and $\mathrm{Y}$ directions. The step size utilized is much larger than the resolution of the stages; the nanopositioner has $1 \mathrm{~nm}$ resolution and the micropositioner has $50 \mathrm{~nm}$ resolution and repeatability of less than $100 \mathrm{~nm}$. In order to explore the dynamics across the RTIL microdroplet, a region of interest (ROI) was chosen such that interfacial environments and bulk environments could be imaged. 2D IR spectra were collected as a function of waiting time ( $\left.T_{w}\right)$ at $2 \mu \mathrm{m}$ steps throughout the ROI. Thus, the ROI was spatially oversampled given the FWHM diameter of the emitted third order signal. A bright field image of the sample was collected using a visible beam splitter and a $2 \mathrm{x}$ imaging lens to project the image onto a visible camera. We correlated the position of the bright field image with the position at which the 2D IR spectra are collected by maximizing the IR throughput of the probe and pump beams through a pinhole at the focal plane. We then imaged the pinhole onto the visible camera and utilized the pinhole location as the location of our 2D IR collection. Under these conditions the image shown in Figure 3a was acquired in 120 minutes; thus, producing an acquisition rate of 1984 individual 2D IR spectra per minute. A representative 2D IR spectrum collected in these experiments is shown in Figure 3b. 
Results and Discussion. Tricyanomethanide (TCM-), is a trigonal planar anion, is evenly dispersed within the microdroplet, and is used as a vibrational probe in the experiments presented in this work. In order to characterize the linear IR response of TCM- in a microdroplet, a FTIR microscope image of an EmimBF4/EmimTCM RTIL microdroplet in silicone oil was collected and is shown in Figure 2a. The linear IR spectrum of TCM- exhibits an absorption band in the 2100-2200 cm-1 region with a peak appearing at $2164 \mathrm{~cm}-1$ corresponding to the degenerate asymmetric stretching vibrations of TCM. The chemical map shown in Figure 2a is produced by integrating the intensity of the TCM- peak centered at $2164 \mathrm{~cm}-1$. The FTIR spectra extracted from different locations across the droplet are compared in Figure 2b; the locality of the spectra are indicated by the points denoted in Figure 2a. The chemical map generated from FTIR microscopy shows the TCM- probe to be homogenously distributed throughout the droplet, as there are no fluctuations of the intensity of the peak associated with the TCM- throughout the microdroplet; the peak intensity only decreases at the interface of the RTIL and the silicon oil. The FTIR spectra at each point have the same FWHM linewidth of $15.5 \pm 0.04 \mathrm{~cm}-1$ and center frequency of $2164.0 \pm$ $0.07 \mathrm{~cm}-1$. Thus, any underlying solute-solvent interactions in the RTIL microdroplet remain hidden in FTIR microscopy. However, utilizing 2D IR microscopy, such interactions can be extracted by studying the chemical dynamics across the microdroplet. 

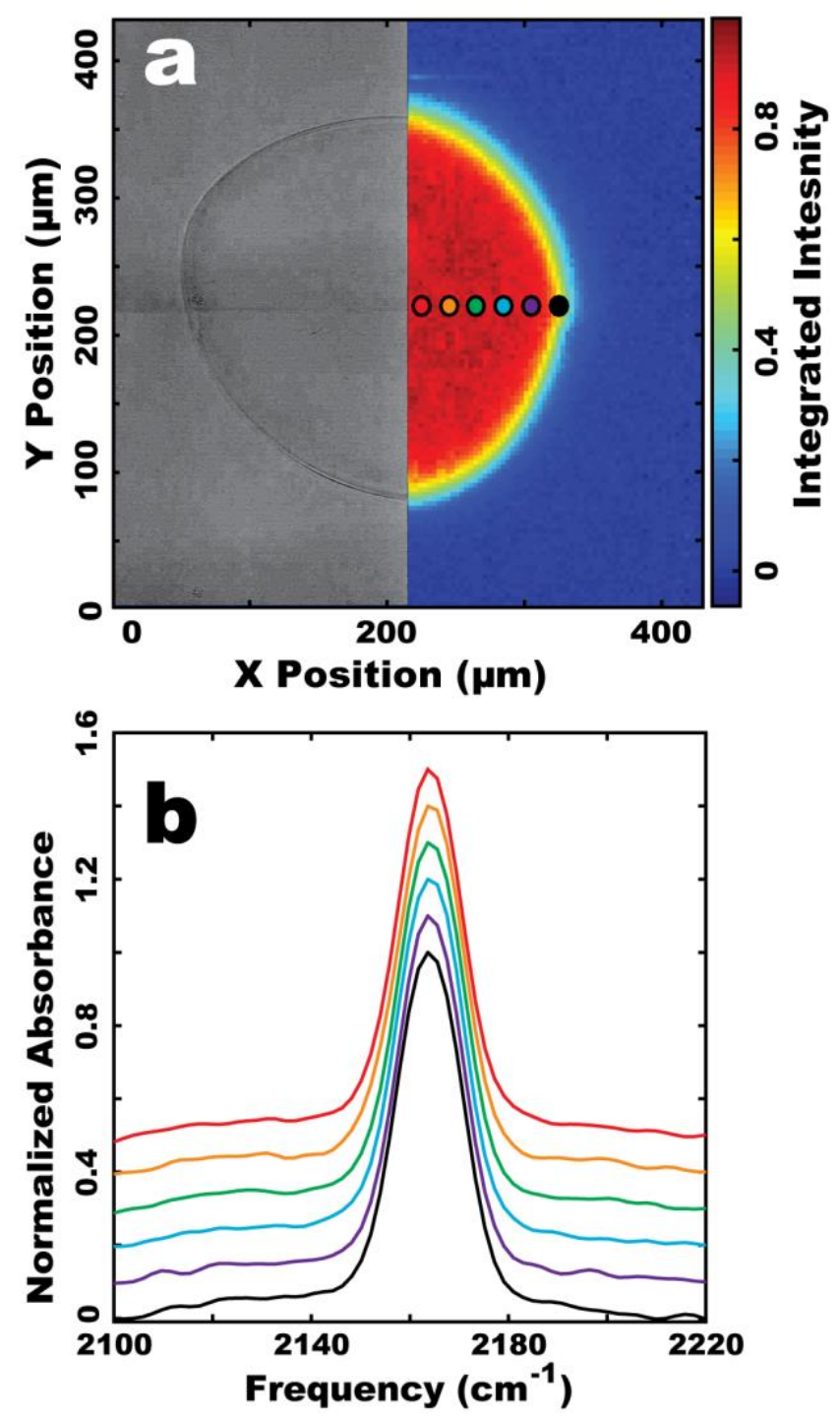

Figure 2. (a) Bright-field and FTIR microscopy image of the RTIL microdroplet. The intensity values on the chemical map are generated by integrating the FTIR spectra of each point from 2146 $\mathrm{cm}_{-1}$ to $2186 \mathrm{~cm}_{-}$. (b) Comparison of FTIR spectra extracted for the set of points across the droplet, indicated in the chemical map above. The FTIR spectra are offset for ease of viewing.

The 2D IR image encompassing the entire RTIL microdroplet was produced by collecting 2D IR spectra at $25 \mu \mathrm{m}$ intervals along the $\mathrm{X}$ and $\mathrm{Y}$ axes (laboratory frame) by scanning the sample stage with a nanopositioner. In Figure 3a, the bright field image of the RTIL microdroplet is shown, where the points overlaid with the image indicate the spatial positions of the collected 2D IR spectra. Figure $3 \mathrm{~b}$ presents a 2D IR spectrum representative of the bulk environment position, 
indicated by the white circle in Figure 3a. The blue contours represent signals generated by $\mathrm{v}=0 \rightarrow 1$ transitions, while the yellow-red contours arise from $\mathrm{v}=1 \rightarrow 2$ transitions. The $\mathrm{v}=1 \rightarrow 2$ band is red shifted due to the vibrational anharmonicity of the asymmetric stretching vibrations of the TCM-. The intensity of points in the 2D IR image are determined by integrating the peak associated with the $\mathrm{v}=1 \rightarrow 2$ transition in the $2 \mathrm{D}$ IR spectrum collected at each point. In this image the spectra collected were each an average of 500 2D IR spectra at a spectral acquisition rate of 1984 individual spectra per minute. It is important to note that a comparison was performed for data acquired under different averaging conditions at the position representative of the bulk environment. When comparing a single 2D IR spectrum, a spectrum generated by averaging 25 spectra, and 250 spectra it is notable that even with no averaging the signal can be observed and the noise floor in the data does not overwhelm the spectral shape. Therefore, in future experiments one may choose to utilize different averaging schemes and use the 1984 spectra per minute acquisition rate to best suit the purpose of the experiment. The use of a Yb-based, $100 \mathrm{kHz}$ laser system also allowed us to utilize pulse energies of $70 \mathrm{~nJ} /$ pulse, which were much lower than those typically used for 2D IR spectroscopy performed with Ti:sapphire systems at lower repetition rates. These signal-to-noise ratios and lower pulse intensities are a result of the use of a $\mathrm{Yb}$ laser source for the $100 \mathrm{kHz}$ system instead of the Ti:sapphire systems typically used for 2D IR. It has been recently demonstrated that Ti:sapphire systems exhibit higher noise at all frequencies and has a significantly shorter shot to shot correlation time than $\mathrm{Yb}$ based gain media.36,37 

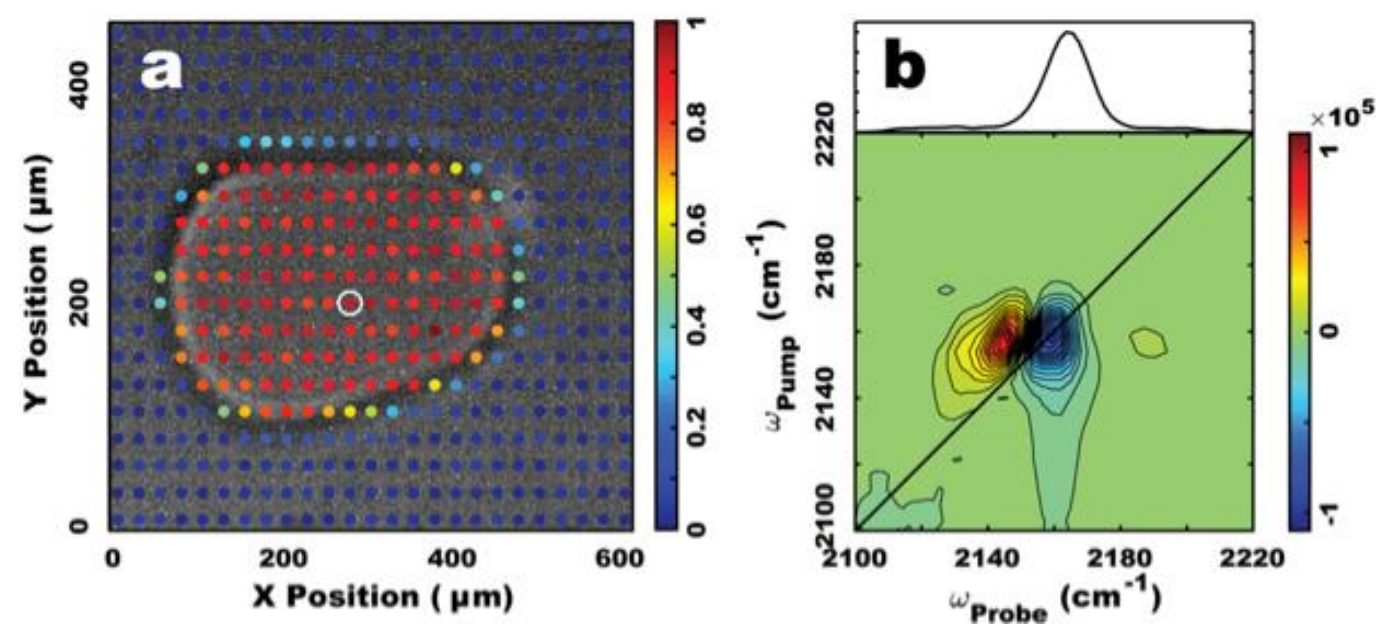

Figure 3. Bright field and 2D IR image of a RTIL microdroplet cast in silicon oil (a). A representative 2D IR spectrum collected across the RTIL microdroplet (b). The white circle in (a) indicates the position in the microdroplet where this 2D IR spectrum was collected. A linear IR spectrum collected in the RTIL microdroplet is shown above the 2D IR spectrum for reference.

The high time resolution inherent in 2D IR spectroscopy allows one to examine the response of a vibrational probe to its solvation environment and directly measure the time scales of motion related to the solvent molecules.38-43 Using both the time and spatial resolution of 2D IR microscopy, dynamics information of different solvation regions across the microdroplet can be examined. In these experiments, the time delay ( $\left.\mathrm{T}_{\mathrm{w}}\right)$ between the second and third pulses is changed and the solvent environment is allowed to evolve after pumping the system. A 2D IR microscopy experiment aimed at extracting the vibrational dynamics of the TCM- probe was performed over a ROI of the RTIL microdroplet. The ROI is indicated by the white box in Figure 4a; 2D IR spectra were collected as a function of $\mathrm{T}_{\mathrm{w}}$ and were collected at $2 \mu \mathrm{m}$ intervals throughout the ROI. It is observed that the diagonal elongation decreases and the $2 \mathrm{D}$ spectra become gradually spherical due to spectral diffusion as $\mathrm{T}_{\mathrm{w}}$ increases. 

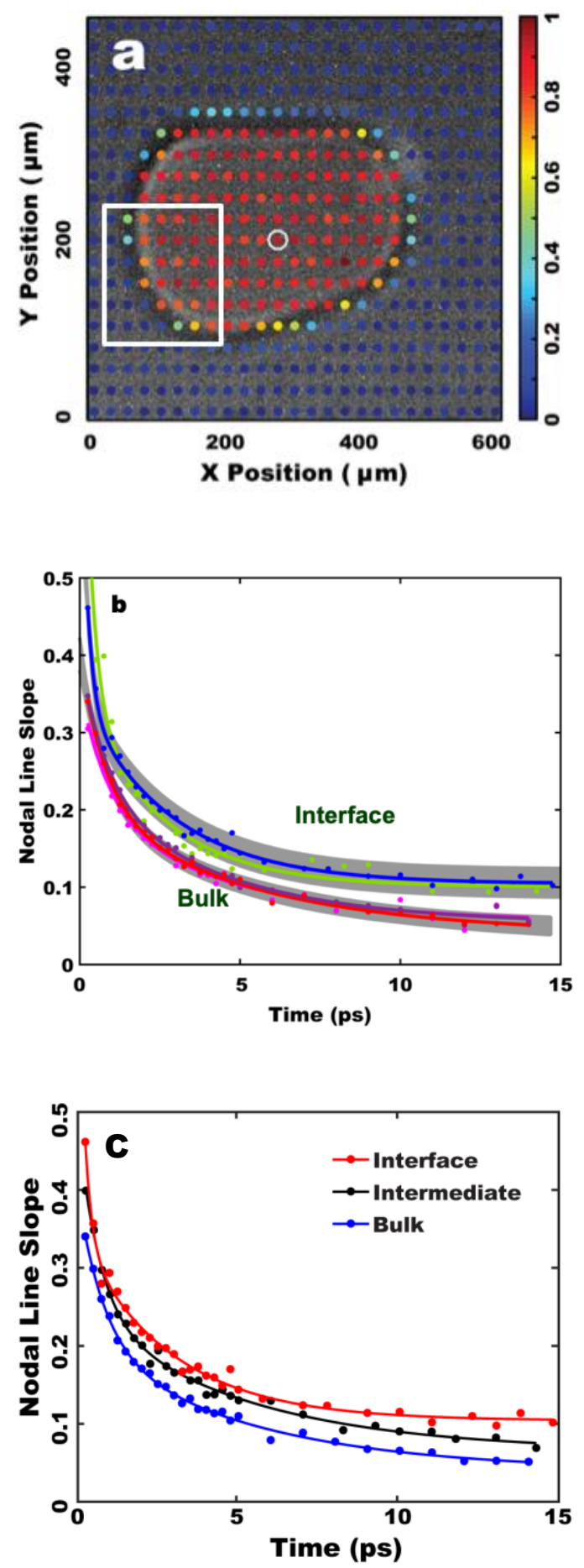

Figure 4. (a)Chemical map of TCM- in the RTIL microdroplet generated from the 2D IR imaging experiments with white box indicating the ROI chosen for $T_{w}$-dependent $2 \mathrm{D}$ IR experiments. (b) Comparison of NLS curves extracted from two points in the interfacial region (blue and green curves) and three points from the bulk region (red, purple, and magenta curves); the 95\% confidence intervals for data from each region is shown as the grey areas. (c)Comparison of NLS decay data extracted for spatial points representative of the bulk environment, the interfacial 
environment, and the chemical environment between the bulk and interfacial regions. In panels (b) and (c) the points are the experimentally measured values and the lines indicate the biexponential fits to the data.

We performed nodal line slope analysis (NLS)41-45 of the collected 2D IR spectra to characterize the spectral diffusion dynamics of TCM- in an RTIL microdroplet. Parameters such as correlation times and the amplitudes of the frequency fluctuation correlation functions (FFCFs) can be used to characterize the spectral diffusion dynamics.23,43,44 In Figure 4c, the NLS values are plotted as a function of $\mathrm{T}_{\mathrm{w}}$, thus depicting the vibrational dynamics from different regions of the microdroplet - the interfacial environments, bulk environments, and environments that are intermediate between the interface and bulk. The slope values decay as $\mathrm{T}_{\mathrm{w}}$ increases. It can be seen in Figure $4 b$ that the decay curves at bulk and interfacial regions are significantly different. Two NLS curves representative of the interfacial region are plotted in blue and green, whereas three NLS curves representative of the bulk region are plotted in red, magenta, and pink; the $95 \%$ confidence intervals of the fits to the points comprising these NLS decay curves are indicated by the gray shadow. In Figure 4c, the NLS decay curves at a representative intermediate point (black) is compared to NLS decay curves of the interfacial region (red) and bulk region (blue). By inspection of the curves it is apparent the intermediate region exhibits a combined behavior of the bulk and the interface. In order to develop a quantitative picture of the chemical dynamics within the RTIL microdroplet, the decays were fit to a biexponential function following the model,

$$
C\left(T_{W}\right)=a_{1} \exp \left(-T_{W} / \tau_{1}\right)+a_{2} \exp \left(-T_{W} / \tau_{2}\right)+b
$$

The biexponential fit of the bulk region gives a $\tau_{1}=810 \pm 100 \mathrm{fs}$ and a $\tau_{2}=5.2 \pm 1.0 \mathrm{ps}$. In contrast, the interfacial region produces a $\tau_{1}=210 \pm 50 \mathrm{fs}$ and a $\tau_{2}=2.8 \pm 0.3 \mathrm{ps}$. Therefore, the time components indicate that the signals encoding the vibrational dynamics decay markedly faster at the interfacial region than in the bulk environments within the RTIL microdroplets. The NLS 
decay curve representative of the intermediate region was collected at a position $5 \mu \mathrm{m}$ to the interior of the microdroplet. This intermediate region exhibits a combination of bulk and interfacial behaviors, producing $\tau_{1}=650 \pm 100 \mathrm{fs}$ and $\tau_{2}=5.0 \pm 0.6 \mathrm{ps}$. These results suggest that the structural rearrangement of the RTIL occurs on different time scales at the interface compared to the interior region of the microdroplet.

In the environment of the microdroplet, the NLS decays at short time scales are most likely caused by ultrafast fluctuation of the first solvation shell of the ionic polar region, and the long time constant arises as a result of longer length scale motions involving dynamics from ionic and partly from nonionic domains, in agreement with earlier alkyl chain length dependent studies of RTILs.22,46 However, the differences in the short time constants of the NLS decays from the interfacial region and the bulk regions indicate differences in how the TCM- is able to sample the structural configurations available in these regions of the ionic liquid. The differences in the NLS time constants from the interfacial region and the bulk region indicate that the rotational motion of the TCM- is restricted in the interfacial region as compared to the bulk region. In other words, the rotational motion of the TCM- is slower in the interfacial region than in the bulk region of the RTIL microdroplet.

The chemical dynamics observed in this investigation reveal interesting characteristics of RTILs that are comparable to other immiscible chemical systems. For example, previous simulation studies of water-organic nonpolar liquids $47-49$ and the water-air interface 50 have reported that the structural relaxation dynamics and reorientation dynamics of interfacial water molecules are slower than the bulk water molecules. In the present study, the dynamics of structural relaxation in the microdroplet interfacial region is expected to be slower than the bulk region, taking into consideration the contribution of the nanoscale silicone oil-RTIL interface. The silicone oil is 
hydrophobic, inert and immiscible with the RTIL (EmimBF4/EmimTCM), and previously it has been used in microfluidics to provide a barrier against molecular cross talk between adjacent droplets. 52,53 The static dielectric constant $(\varepsilon 0 \sim 2.4)$ and viscosity $(\eta \sim 50 \mathrm{cP})$ of the silicone oil are significantly different from the RTIL investigated here $54, \varepsilon_{0} \sim 14$ and $\eta \sim 28 \mathrm{cP}$, respectively. Thus, the Si-O unit of the oil may have a minor interaction with the ionic component of the RTIL; however, the contribution of such an interaction on spectral diffusion is mostly insignificant. However, the presence of the interface restricts the rotational motion of TCM- in the RTIL which is observed by the faster time constant with which the third order signal decays at the interface. In addition, the static offset observed in the NLS curves from the interfacial region reveal the fact that the TCM- probe molecules are moving too slow to experience all possible structural configurations and thus, local environments in the RTIL.

Spectral diffusion dynamics in RTILs have been previously studied using linear vibrational probes thiocyanate $(\mathrm{SCN}-)$ and selenocyanate $(\mathrm{SeCN}-)$; the dynamics were observed to be slower than the present experiment.21-26,46,55 For example, the fast time components were a few ps and the long time components were a few 10s of ps. In the present study, the structure of the vibrational probe, TCM-, is trigonal planar and the charge density of the probe is smaller than the linear anionic vibrational probes, $\mathrm{SCN}$ - or $\mathrm{SeCN}$-, owing to the delocalization of charge over the three $\mathrm{CN}$ groups. It is possible the slow spectral diffusion of a linear anionic probe in RTILs arises because of the higher charge density compared to the trigonal planar TCM- anion. In addition, because the 2D IR spectra were collected in a cross-polarization geometry in this work, the measurements are sensitive to the fast time components of the dynamics, a point that will be discussed further in future work. 
It is clear from the nodal line slope decays presented in Figure 4b-c that the homogeneous contribution and spectral diffusion followed by a static inhomogeneity constitute the frequency fluctuations of the asymmetric CN stretching of TCM- in the RTIL microdroplet. The homogeneous contribution is a large motionally narrowed component, which arises from very fast fluctuations due to low frequency librations56 in the RTIL and dominates the spectral line shape. The slope values at the initial waiting time indicate that the amount of homogeneous contribution increases moving from the interfacial environment to the bulk environment. Moreover, at the interfacial region, the 2D IR spectra are more inhomogeneously broadened than the bulk at all waiting times - a result nicely reflected in the decays of the nodal line slopes. Looking at the static offset of the different regions, the bulk and the interfacial regions show an offset of 0.05 and 0.10 , respectively. The larger static offset at the interface is an indicator of greater static inhomogeneous contributions, which exhibit very slow relaxation of the FFCF in our experimental time window. The larger inhomogeneous contribution to the frequency fluctuation appears as a result of anisotropic orientation of ions in the RTIL microdroplet. This effect is more dramatic at the interface where the motion of the probe molecules is restricted, resulting in the shorter time constants in the NLS decays from the interface which also reflects faster spectral diffusion dynamics at the interface. Nevertheless, further study is required to determine the mechanistic details of spectral diffusion in the RTIL microdroplet. The observation of distinctly different dynamic behaviors, along with homogeneous and inhomogeneous contributions to the frequency fluctuations underscore the importance of solute-solvent interactions in studying dynamics across the multitude of environments present in a RTIL microdroplet. Therefore, the present results of this 2D IR microscopy investigation are of high importance to the understanding of the RTIL microdroplet chemistry. 
The bulk environment and interfacial environment are two extremes in the RTIL microdroplet. However, it is important to consider the intermediate region between the bulk and interfacial environments in the RTIL microdroplet. The behavior in the intermediate region of the RTIL microdroplet can be discussed in the light of interactions of various length scales proposed in RTILs. RTIL structures at interfaces and in confinement have been extensively studied by experiment and simulation.23,28-31,57-65 It has been found that the presence of the interface can influence the local charge ordering, thus changing the structural properties of RTILs. While most studies report that such interactions occur on a few nanometer length scale, there are some recent experimental observations that are in stark contrast. Recent experimental observations indicate that the interface can induce much longer ranged effects that span several 10 s of nanometers to a few micrometers in length scale.23,30,31 For example, very recent 2D IR spectroscopic studies of supported ionic liquid membranes with pore sizes of $\sim 300 \mathrm{~nm}$ showed a significant retardation of RTIL structural dynamics compared to the bulk phase.23 Additional 2D IR spectroscopy experiments on RTIL thin films reveal similar changes in dynamic behaviors as the film is made thicker. Another spectroscopic experiment reported the transition of TFSI anion-based RTIL films into long-ranged ordered structures that persist over $2 \mu \mathrm{m}$ when the films are formed by applying a slow shear.30 In the present work, the chemical dynamics and spectral line shapes observed are a combination of bulk-like and interface-like as deep as $5 \mu \mathrm{m}$ to the interior of the microdroplet. Although the apparent spatial resolution of our technique is not small enough to observe such phenomena, because the 2D IR signal depends on the square of the intensity, it is able to provide spatial resolution that is better than the FWHM beam diameters suggest, similar in fashion to twophoton fluorescence microscopy.66,67 Therefore, the perturbation of the interface to the bulk environment in this system is long-ranged. In order to explore the possible length scales over 
which the interface may contribute or perturb the bulk dynamics of the system, we model the NLS data with several different functions.

The changes in amplitudes, time constants, and offsets can be modeled to determine how the influence of the dynamical behaviors at the interface might propagate across this IL microstructure. In order to model the data appropriately we must take into account the spatial distribution of the signal intensity for the third-order 2D IR signal, the dynamics as a function of sample location and the contribution from each spatial region within the beam profile. The microscopy data was modeled by first considering the spatial distribution of the signal intensity for the $2 \mathrm{D}$ signal. The self-heterodyned signal for the square law detection is given by,

$$
S_{\text {self heterodyned }} \alpha\left|P^{3}+E_{\text {probe }}\right|^{2}
$$

Where $S_{\text {self heterodyned }}$ is the detected colinear signal, $P^{3}$ is the third order polarization and $E_{\text {probe }}$ is the probe field. $P^{3}$ is proportional to the product of the three incident fields and the dominating signal term is proportional to the intensity of both the pump and probe lines.

$$
S_{\text {self heterodyned }} \alpha I_{\text {pump }} I_{\text {probe }}
$$

Where $I_{\text {pump }}$ is the pump intensity and $I_{\text {probe }}$ is the probe intensity and the two pump fields are considered to have equal amplitude. The spatial distribution of the signal intensity was modeled using a probe intensity with a FWHM of $14 \mu \mathrm{m}$ in the horizontal direction and $20 \mu \mathrm{m}$ in the vertical direction and pump beam with a $20 \mu \mathrm{m}$ FWHM in both directions. The product of the pump and probe intensity profiles results in a signal distribution that was integrated vertically to determine the signal profile in the horizontal direction, which is the direction the sample is displaced as it is moved away from the interface region. The resulting horizontal signal distribution has a FWHM of $11.4 \mu \mathrm{m}$. In order to model the collected signal, the dynamics as function of sample location and the contribution from each spatial region of the beam profile need to be considered. The 2D 
signal is assumed to be comprised of multiple environments along the horizontal direction away from the interface. Each region's NLS curve is modeled as a biexponential with an offset.

$$
C_{N L S(X)}=\alpha_{1 X} \exp \left(\frac{-T_{W}}{\tau_{1 X}}\right)+\alpha_{2 X} \exp \left(\frac{-T_{W}}{\tau_{2 X}}\right)+\text { offset }_{X}
$$

Where $\mathrm{X}$ is the distance from the interface and $T_{w}$ is the waiting time. The collected signal contains the signal from each spatial region weighted by the signal intensity in that region according to,

$$
C_{N L S}=\sum_{X} \beta_{X}\left[C_{N L S(X)}\right]
$$

where, $\beta_{X}$ is the weighting factor at position $\mathrm{X}$ from the spatial distribution of the signal intensity. We assume the dynamics change continuously from interface to bulk dynamics with an unknown length scale and rate of change. No signal is generated in the silicon oil. A cartoon of the model is shown in Fig. 5a.
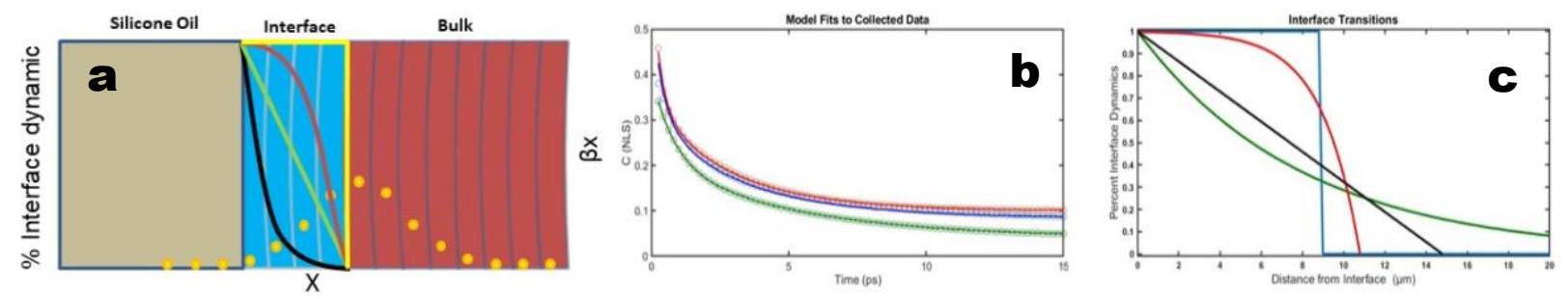

Figure 5. (a)Cartoon model of all sample components. The shapes of the four spatial models used to fit the experimental NLS decay curves from three points collected laterally across the ROI of the IL microdroplet. (b)Fits (lines) by the models to the experimental NLS data (open circles) from the interface (red), a point intermediate to the interface and bulk regions (blue), and the bulk region (green). (c) The functions describing the transition from interfacial dynamics to bulk dynamics as a function of distance from the interface by each model used: binary (blue), linear (black), exponential (green), and reverse exponential (red). 
The total IL microdroplet sample consists of silicon oil, interface and bulk regions and are shown as brown, blue, and red blocks in the cartoon in Fig. 5a, respectively. The interface and bulk are divided into spatial regions that are $1 \mu \mathrm{m}$ slabs with their individual $C_{N L S(X)}$ and $\beta_{X}$. We consider several possible transitions from the interface to bulk dynamics in which the $\alpha_{i} \mathrm{~s}$ and $\tau_{i}$ s change from the interface values to the bulk values over the transition length. The first model is a binary model with an interface region and a bulk region shown in yellow in Fig. 5a. The interface region is taken to be homogeneous and the length of the interface region shown in blue in Fig. 5c. Only interface and bulk parameters are needed in the binary model and the interface length is adjusted to fit the NLS curves extracted from three points across the RTIL microdroplet. The weighted contributions depending on beam position determine the $C_{N L S}$. We have fit the NLS decay curves with a binary model, a linear model, an exponential model, and a reverse exponential model. The shapes of the models are shown in Fig. 5c and an example of the fits to the data are shown in Figure $5 \mathrm{~b}$. The experimental data generating the NLS curves for three points in the ROI, interfacial region (red circles), bulk region (green circles), and an intermediate point (blue circles) are fit according to the spatial models briefly described above. Each spatial model produces a length scale over which the dynamics transition from interfacial behavior to bulk behavior. The weighting factors for the percent of the interface dynamic through the transition region are shown in Figure 5c. Using the binary model an interface region of $9 \mu \mathrm{m}$ gives the best fit to the experimental data. The linear model is best fit with a $15 \mu \mathrm{m}$ interface length. The exponential fit gives the best fit when the transition length is $40 \mu \mathrm{m}$ with a sigma of $5 \mu \mathrm{m}$ and the reverse exponential best fit produces a transition length of $12 \mu \mathrm{m}$ and a sigma of $3 \mu \mathrm{m}$. At this time, we cannot distinguish which model truly describes the spatial dependence of the dynamics measured. However, we can consider which model is the most physically reasonable with intuition. For 
example, a $9 \mu \mathrm{m}$ thick interface as determined using the binary model is non-physical. Perhaps the most reasonable model is the reverse exponential model in which the contribution to the dynamics from the interface contributes significantly for approximately $5 \mu \mathrm{m}$, but then transitions continuously to bulk dynamics in a rapid manner. The lengths over which these data suggest the interface perturbs the dynamic behaviors across the IL microdroplet are long, but not unreasonable given the long-range ordering recently observed in IL films, supported IL membranes, and ILs under shear.

\section{Conclusions.}

In summary, we have for the first time, spatially resolved ultrafast chemical dynamics existing in a RTIL microdroplet. Utilizing a 2D IR microscope based on a high repetition rate mid-IR laser system allowed a fast acquisition rate of 1984 spectral points per minute to be achieved. The advances in 2D IR microscopy demonstrated in this work provide the opportunity to explore the chemical dynamics in the inherently complex environment of a RTIL microdroplet.

2D IR microscopy experiments were performed across the RTIL microdroplet and a region of interest was selected to include the bulk (interior) environment, interfacial environments, and intermediate regions between the interfacial and the bulk environments. A series of waiting time, $\mathrm{T}_{\mathrm{w}}$, measurements made throughout the region of interest revealed the complex nature of the chemical dynamics in RTIL microdroplets; the homogeneous and inhomogeneous contributions to the vibrational frequency fluctuations of TCM- are used to probe the nature of the chemical dynamics present in the RTIL microdroplet. Our study determined markedly faster decays in the vibrational dynamics at the droplet interface in comparison to the bulk or interior of the microdroplet. Moreover, the dynamics observed in the regions intermediate to the bulk and 
interfacial environments are a combination of bulk-like dynamics and interfacial-like dynamics. Functional models were used to explore how the dynamics evolve across the microdroplet from the interfacial region to the bulk region of the microdroplet. These models shed light on the potential impact of long-range interactions that exist in these RTIL microdroplets. The results from this first ultrafast study of microdroplet chemical dynamics are of great importance to understand the striking features of these systems and provide a basis for further investigation. Finally, this work demonstrates the potential power of 2D IR microscopy performed with fast acquisition rates for revealing chemical dynamics in complex environments that are hidden in other chemical imaging techniques.

\section{AUTHOR INFORMATION}

\section{Corresponding Author}

*Email: amber.krummel@colostate.edu

\section{Author Contributions}

The manuscript was written through contributions of all authors. All authors have given approval to the final version of the manuscript. $\$$ K.M.T. and B.G. are co-first authors and contributed equally to this work.

\section{Funding Sources}

This research was supported by BP Exploration Operating Company Ltd., the Alfred P. Sloan Foundation, and the Department of Energy (Grant DE-SC0016137). 


\section{ACKNOWLEDGMENT}

The authors appreciate the financial support of this work by BP Exploration Operating Company Ltd., the Alfred P. Sloan Foundation, and the Department of Energy (Grant DE-SC0016137).

\section{ABBREVIATIONS}

RTIL, room temperature ionic liquid; TCM-, tricyanomethanide; 2DIR, two-dimensional infrared; TFSI, bis(trifluoromethane)sulfonimide.

\section{REFERENCES}

(1) Bain Ryan M.; Sathyamoorthi Shyam; Zare Richard N. "On-Droplet” Chemistry: The Cycloaddition of Diethyl Azodicarboxylate and Quadricyclane. Angew. Chem. Int. Ed. 2017, 56 (47), 15083-15087.

(2) Lee, J. K.; Banerjee, S.; Nam, H. G.; Zare, R. N. Acceleration of Reaction in Charged Microdroplets. Q. Rev. Biophys. 2015, 48 (4), 437-444.

(3) Li Yafeng; Yan Xin; Cooks R. Graham. The Role of the Interface in Thin Film and Droplet Accelerated Reactions Studied by Competitive Substituent Effects. Angew. Chem. Int. Ed. 2016, 55 (10), 3433-3437.

(4) Yan Xin; Cheng Heyong; Zare Richard N. Two-Phase Reactions in Microdroplets Without the Use of Phase-Transfer Catalysts. Angew. Chem. Int. Ed. 2017, 56 (13), 3562-3565.

(5) Nam, I.; Lee, J. K.; Nam, H. G.; Zare, R. N. Abiotic Production of Sugar Phosphates and Uridine Ribonucleoside in Aqueous Microdroplets. Proc. Natl. Acad. Sci. 2017, 114 (47), 1239612400. 
(6) Fallah-Araghi, A.; Meguellati, K.; Baret, J.-C.; Harrak, A. E.; Mangeat, T.; Karplus, M.; Ladame, S.; Marques, C. M.; Griffiths, A. D. Enhanced Chemical Synthesis at Soft Interfaces: A Universal Reaction-Adsorption Mechanism in Microcompartments. Phys. Rev. Lett. 2014, 112 (2), 028301.

(7) Barikbin, Z.; Rahman, M. T.; Parthiban, P.; Rane, A. S.; Jain, V.; Duraiswamy, S.; Lee, S. H. S.; Khan, S. A. Ionic Liquid-based Compound Droplet Microfluidics for "on-drop” Separations and Sensing. Lab. Chip 2010, 10 (18), 2458-2463.

(8) Lazarus, L. L.; Riche, C. T.; Marin, B. C.; Gupta, M.; Malmstadt, N.; Brutchey, R. L. TwoPhase Microfluidic Droplet Flows of Ionic Liquids for the Synthesis of Gold and Silver Nanoparticles. ACS Appl. Mater. Interfaces 2012, 4 (6), 3077-3083.

(9) Lazarus, L. L.; Yang, A. S.-J.; Chu, S.; Brutchey, R. L.; Malmstadt, N. Flow-focused Synthesis of Monodisperse Gold Nanoparticles Using Ionic Liquids on a Microfluidic Platform. Lab. Chip 2010, 10 (24), 3377-3379.

(10) Malankowska, M.; Martins, C. F.; Rho, H. S.; Neves, L. A.; Tiggelaar, R. M.; Crespo, J. G.; Pina, M. P.; Mallada, R.; Gardeniers, H.; Coelhoso, I. M. Microfluidic Devices as Gas - Ionic Liquid Membrane Contactors for CO2 Removal from Anaesthesia Gases. J. Membr. Sci. 2018, $545,107-115$.

(11) Canongia Lopes, J. N. A.; Pádua, A. A. H. Nanostructural Organization in Ionic Liquids. J. Phys. Chem. B 2006, 110 (7), 3330-3335. 
(12) Samanta, A. Dynamic Stokes Shift and Excitation Wavelength Dependent Fluorescence of Dipolar Molecules in Room Temperature Ionic Liquids. J. Phys. Chem. B 2006, 110 (28), 1370413716.

(13) Jr, E. W. C.; Margulis, C. J.; Maroncelli, M.; Wishart, J. F. Ionic Liquids: Structure and Photochemical Reactions. Annu. Rev. Phys. Chem. 2011, 62 (1), 85-105.

(14) Egorova, K. S.; Gordeev, E. G.; Ananikov, V. P. Biological Activity of Ionic Liquids and Their Application in Pharmaceutics and Medicine. Chem. Rev. 2017, 117 (10), 7132-7189.

(15) Tadesse, H.; Luque, R. Advances on Biomass Pretreatment Using Ionic Liquids: An Overview. Energy Environ. Sci. 2011, 4 (10), 3913-3929.

(16) Watanabe, M.; Thomas, M. L.; Zhang, S.; Ueno, K.; Yasuda, T.; Dokko, K. Application of Ionic Liquids to Energy Storage and Conversion Materials and Devices. Chem. Rev. 2017, 117 (10), 7190-7239.

(17) Lin, R.; Taberna, P.-L.; Fantini, S.; Presser, V.; Pérez, C. R.; Malbosc, F.; Rupesinghe, N. L.; Teo, K. B. K.; Gogotsi, Y.; Simon, P. Capacitive Energy Storage from -50 to $100{ }^{\circ} \mathrm{C}$ Using an Ionic Liquid Electrolyte. J. Phys. Chem. Lett. 2011, 2 (19), 2396-2401.

(18) Plechkova, N. V.; Seddon, K. R. Applications of Ionic Liquids in the Chemical Industry. Chem. Soc. Rev. 2007, 37 (1), 123-150.

(19) Smiglak, M.; Metlen, A.; Rogers, R. D. The Second Evolution of Ionic Liquids: From Solvents and Separations to Advanced Materials-Energetic Examples from the Ionic Liquid Cookbook. Accounts Chem. Res. 2007, 40 (11), 1182-1192. 
(20) Baldelli, S. Interfacial Structure of Room-Temperature Ionic Liquids at the Solid-Liquid Interface as Probed by Sum Frequency Generation Spectroscopy. J. Phys. Chem. Lett. 2013, 4 (2), $244-252$.

(21) Tamimi, A.; Fayer, M. D. Ionic Liquid Dynamics Measured with 2D IR and IR PumpProbe Experiments on a Linear Anion and the Influence of Potassium Cations. J. Phys. Chem. B 2016, 120 (26), 5842-5854.

(22) Tamimi, A.; Bailey, H. E.; Fayer, M. D. Alkyl Chain Length Dependence of the Dynamics and Structure in the Ionic Regions of Room-Temperature Ionic Liquids. J. Phys. Chem. B 2016, $120(30), 7488-7501$.

(23) Shin, J. Y.; Yamada, S. A.; Fayer, M. D. Dynamics of a Room Temperature Ionic Liquid in Supported Ionic Liquid Membranes Vs the Bulk Liquid: 2D IR and Polarized IR Pump-Probe Experiments. J. Am. Chem. Soc. 2017, 139 (1), 311-323.

(24) Ren, Z.; Brinzer, T.; Dutta, S.; Garrett-Roe, S. Thiocyanate as a Local Probe of Ultrafast Structure and Dynamics in Imidazolium-Based Ionic Liquids: Water-Induced Heterogeneity and Cation-Induced Ion Pairing. J. Phys. Chem. B 2015, 119 (13), 4699-4712.

(25) Dutta, S.; Ren, Z.; Brinzer, T.; Garrett-Roe, S. Two-dimensional Ultrafast Vibrational Spectroscopy of Azides in Ionic Liquids Reveals Solute-specific Solvation. Phys. Chem. Chem. Phys. 2015, 17 (40), 26575-26579.

(26) Ren, Z.; Ivanova, A. S.; Couchot-Vore, D.; Garrett-Roe, S. Ultrafast Structure and Dynamics in Ionic Liquids: 2D-IR Spectroscopy Probes the Molecular Origin of Viscosity. $J$. Phys. Chem. Lett. 2014, 5 (9), 1541-1546. 
(27) K. Kashyap, H.; J. Hettige, J.; R. Annapureddy, H. V.; J. Margulis, C. SAXS Anti-peaks Reveal the Length-scales of Dual Positive-negative and Polar-apolar Ordering in Roomtemperature Ionic Liquids. Chem. Commun. 2012, 48 (42), 5103-5105.

(28) Amith, W. D.; Hettige, J. J.; Castner, E. W.; Margulis, C. J. Structures of Ionic Liquids Having Both Anionic and Cationic Octyl Tails: Lamellar Vacuum Interface Vs Sponge-Like Bulk Order. J. Phys. Chem. Lett. 2016, 7 (19), 3785-3790.

(29) Paredes, X.; Fernández, J.; Pádua, A. A. H.; Malfreyt, P.; Malberg, F.; Kirchner, B.;

Pensado, A. S. Using Molecular Simulation to Understand the Structure of [C2C1im]+Alkylsulfate Ionic Liquids: Bulk and Liquid-Vapor Interfaces. J. Phys. Chem. B 2012, 116 (48), $14159-14170$.

(30) Anaredy, R. S.; Shaw, S. K. Long-Range Ordering of Ionic Liquid Fluid Films. Langmuir 2016, $32(20), 5147-5154$.

(31) Jurado, L. A.; Kim, H.; Arcifa, A.; Rossi, A.; Leal, C.; Spencer, N. D.; Espinosa-Marzal, R. M. Irreversible Structural Change of a Dry Ionic Liquid Under Nanoconfinement. Phys. Chem. Chem. Phys. 2015, 17 (20), 13613-13624.

(32) Baiz, C. R.; Schach, D.; Tokmakoff, A. Ultrafast 2D IR Microscopy. Opt. Express 2014, $22(15), 18724-18735$.

(33) Ostrander, J. S.; Serrano, A. L.; Ghosh, A.; Zanni, M. T. Spatially Resolved TwoDimensional Infrared Spectroscopy via Wide-Field Microscopy. ACS Photonics 2016, 3 (7), $1315-1323$. 
(34) Luther, B. M.; Tracy, K. M.; Gerrity, M.; Brown, S.; Krummel, A. T. 2D IR Spectroscopy at $100 \mathrm{kHz}$ Utilizing a Mid-IR OPCPA Laser Source. Opt. Express 2016, 24 (4), 4117-4127.

(35) Tracy, K. M.; Barich, M. V.; Carver, C. L.; Luther, B. M.; Krummel, A. T. HighThroughput Two-Dimensional Infrared (2D IR) Spectroscopy Achieved by Interfacing Microfluidic Technology with a High Repetition Rate 2D IR Spectrometer. J. Phys. Chem. Lett. 2016, 7 (23), 4865-4870.

(36) Kearns, N. M.; Mehlenbacher, R. D.; Jones, A. C.; Zanni, M. T. Broadband 2D Electronic Spectrometer Using White Light and Pulse Shaping: Noise and Signal Evaluation at 1 and 100 kHz. Opt. Express 2017, 25 (7), 7869-7883.

(37) Donaldson, P. M.; Greetham, G. M.; Shaw, D. J.; Parker, A. W.; Towrie, M. A 100 kHz Pulse Shaping 2D-IR Spectrometer Based on Dual Yb:KGW Amplifiers. J. Phys. Chem. A 2018, $122(3), 780-787$.

(38) Zheng, J.; Kwak, K.; Chen, X.; Asbury, J. B.; Fayer, M. D. Formation and Dissociation of Intra-Intermolecular Hydrogen-Bonded Solute-Solvent Complexes: Chemical Exchange TwoDimensional Infrared Vibrational Echo Spectroscopy. J. Am. Chem. Soc. 2006, 128 (9), 29772987.

(39) Khalil, M.; Demirdöven, N.; Tokmakoff, A. Coherent 2D IR Spectroscopy: Molecular Structure and Dynamics in Solution. J. Phys. Chem. A 2003, 107 (27), 5258-5279.

(40) Remorino, A.; Korendovych, I. V.; Wu, Y.; DeGrado, W. F.; Hochstrasser, R. M. ResidueSpecific Vibrational Echoes Yield 3D Structures of a Transmembrane Helix Dimer. Science 2011, 332 (6034), 1206-1209. 
(41) Hamm, P.; Zanni, M. Concepts and Methods of 2D Infrared Spectroscopy; Cambridge University Press, 2011.

(42) Koziński, M.; Garrett-Roe, S.; Hamm, P. Vibrational Spectral Diffusion of CN- in Water. Chem. Phys. 2007, 341 (1-3), 5-10.

(43) Roberts, S. T.; Loparo, J. J.; Tokmakoff, A. Characterization of Spectral Diffusion from Two-dimensional Line Shapes. J. Chem. Phys. 2006, 125 (8), 084502.

(44) Kwac, K.; Cho, M. Molecular Dynamics Simulation Study of N-methylacetamide in Water. II. Two-dimensional Infrared Pump-probe Spectra. J. Chem. Phys. 2003, 119 (4), 22562263.

(45) Kuroda, D. G.; Singh, P. K.; Hochstrasser, R. M. Differential Hydration of Tricyanomethanide Observed by Time Resolved Vibrational Spectroscopy. J. Phys. Chem. B 2013, $117(16), 4354-4364$.

(46) Brinzer, T.; Garrett-Roe, S. Temperature and Chain Length Dependence of Ultrafast Vibrational Dynamics of Thiocyanate in Alkylimidazolium Ionic Liquids: A Random Walk on a Rugged Energy Landscape. J. Chem. Phys. 2017, 147 (19), 194501.

(47) Chowdhary, J.; Ladanyi, B. M. Hydrogen Bond Dynamics at the Water/Hydrocarbon Interface. J. Phys. Chem. B 2009, 113 (13), 4045-4053.

(48) Benjamin, I. Hydrogen Bond Dynamics at Water/Organic Liquid Interfaces. J. Phys. Chem. $B$ 2005, 109 (28), 13711-13715.

(49) Chen, M.; Lu, X.; Liu, X.; Hou, Q.; Zhu, Y.; Zhou, H. Retardation of Water Reorientation at the Oil/Water Interface. J. Phys. Chem. C 2015, 119 (29), 16639-16648. 
(50) Ni, Y.; Gruenbaum, S. M.; Skinner, J. L. Slow Hydrogen-bond Switching Dynamics at the Water Surface Revealed by Theoretical Two-dimensional Sum-frequency Spectroscopy. Proc. Natl. Acad. Sci. 2013, 110 (6), 1992-1998.

(51) Van der Post, S. T.; Hsieh, C.-S.; Okuno, M.; Nagata, Y.; Bakker, H. J.; Bonn, M.; Hunger, J. Strong Frequency Dependence of Vibrational Relaxation in Bulk and Surface Water Reveals Sub-picosecond Structural Heterogeneity. Nat. Commun. 2015, 6, 8384.

(52) Schoeler, A. M.; Josephides, D. N.; Sajjadi, S.; Lorenz, C. D.; Mesquida, P. Charge of Water Droplets in Non-polar Oils. J. Appl. Phys. 2013, 114 (14), 144903.

(53) Ohashi, T.; Kuyama, H.; Hanafusa, N.; Togawa, Y. A Simple Device Using Magnetic Transportation for Droplet-based PCR. Biomed. Microdevices 2007, 9 (5), 695-702.

(54) Singh, T.; Kumar, A. Static Dielectric Constant of Room Temperature Ionic Liquids: Internal Pressure and Cohesive Energy Density Approach. J. Phys. Chem. B 2008, 112 (41), $12968-12972$.

(55) Yamada, S. A.; Bailey, H. E.; Tamimi, A.; Li, C.; Fayer, M. D. Dynamics in a RoomTemperature Ionic Liquid from the Cation Perspective: 2D IR Vibrational Echo Spectroscopy. $J$. Am. Chem. Soc. 2017, 139 (6), 2408-2420.

(56) Mou, S.; Rubano, A.; Paparo, D. Broadband Terahertz Spectroscopy of ImidazoliumBased Ionic Liquids. J. Phys. Chem. B 2018.

(57) Santos, C. S.; Baldelli, S. Gas-liquid Interface of Room-temperature Ionic Liquids. Chem. Soc. Rev. 2010, 39 (6), 2136-2145. 
(58) Hayes, R.; Warr, G. G.; Atkin, R. Structure and Nanostructure in Ionic Liquids. Chem. Rev. 2015, 115 (13), 6357-6426.

(59) Bovio, S.; Podestà, A.; Lenardi, C.; Milani, P. Evidence of Extended Solidlike Layering in [Bmim][NTf2] Ionic Liquid Thin Films at Room-Temperature. J. Phys. Chem. B 2009, 113 (19), 6600-6603.

(60) Hayes, R.; Borisenko, N.; Tam, M. K.; Howlett, P. C.; Endres, F.; Atkin, R. Double Layer Structure of Ionic Liquids at the $\mathrm{Au}(111)$ Electrode Interface: An Atomic Force Microscopy Investigation. J. Phys. Chem. C 2011, 115 (14), 6855-6863.

(61) Mezger, M.; Ocko, B. M.; Reichert, H.; Deutsch, M. Surface Layering and Melting in an Ionic Liquid Studied by Resonant Soft X-ray Reflectivity. Proc. Natl. Acad. Sci. 2013, 110 (10), $3733-3737$.

(62) Antelmi, D. A.; Kékicheff, P.; Richetti, P. The Confinement-Induced Sponge to Lamellar Phase Transition. Langmuir 1999, 15 (22), 7774-7788.

(63) Sieffert, N.; Wipff, G. Ordering of Imidazolium-Based Ionic Liquids at the $\alpha$-Quartz(001) Surface: A Molecular Dynamics Study. J. Phys. Chem. C 2008, 112 (49), 19590-19603.

(64) Atkin, R.; Warr, G. G. Structure in Confined Room-Temperature Ionic Liquids. J. Phys. Chem. C 2007, 111 (13), 5162-5168.

(65) Coasne, B.; Viau, L.; Vioux, A. Loading-Controlled Stiffening in Nanoconfined Ionic Liquids. J. Phys. Chem. Lett. 2011, 2 (10), 1150-1154.

(66) Denk, W.; Strickler, J. H.; Webb, W. W. Two-photon Laser Scanning Fluorescence Microscopy. Science 1990, 248 (4951), 73-76. 
(67) Zipfel, W. R.; Williams, R. M.; Webb, W. W. Nonlinear Magic: Multiphoton Microscopy in the Biosciences. Nat. Biotechnol. 2003, 21 (11), 1369-1377. 